\title{
DE-FG07-96ER 14723 \\ COLLABORATIVE RESEARCH: HYDROGEOLOGICAL-GEOPHYSICAL METHODS FOR SUBSURFACE SITE CHARACTERIZATION
}

\section{March 1, 2000}

Gary Mavko

Department of Geophysics, Stanford University, CA 94305.

Phone: 650-723-9438; email: gary@pangea.stanford.edu

\author{
Yoram Rubin \\ Department of Civil \\ Engineering \\ University of California \\ Berkeley, California 94720 \\ Telephone: (510) 6422282 \\ FAX: (510) 6427476 \\ rubin@arimor.ce.berkeley.edu
}

\author{
Frank Morrison \\ Dept. of Material Science \\ and Mineral Engineering \\ University of California \\ Berkeley, California 94720 \\ Telephone: (510) 6423157 \\ FAX : (510) 6423805 \\ hfmengeo@garnet.berkeley.edu
}

\author{
Jamie Rector \\ Dept. of Material Science \\ and Mineral Engineering \\ University of California \\ Berkeley, California 94720 \\ Telephone: 5106437820 \\ FAX: 5106423805 \\ rector@merit.berkeley.edu
}

Over the course of the project four graduate students and one post-doc were involved, at least part-time, in the research effort.

Summary. The significance of this project is that it addresses the issue of site characterization: not only is this issue a very significant budget item in the site clean-up effort, but also it is now

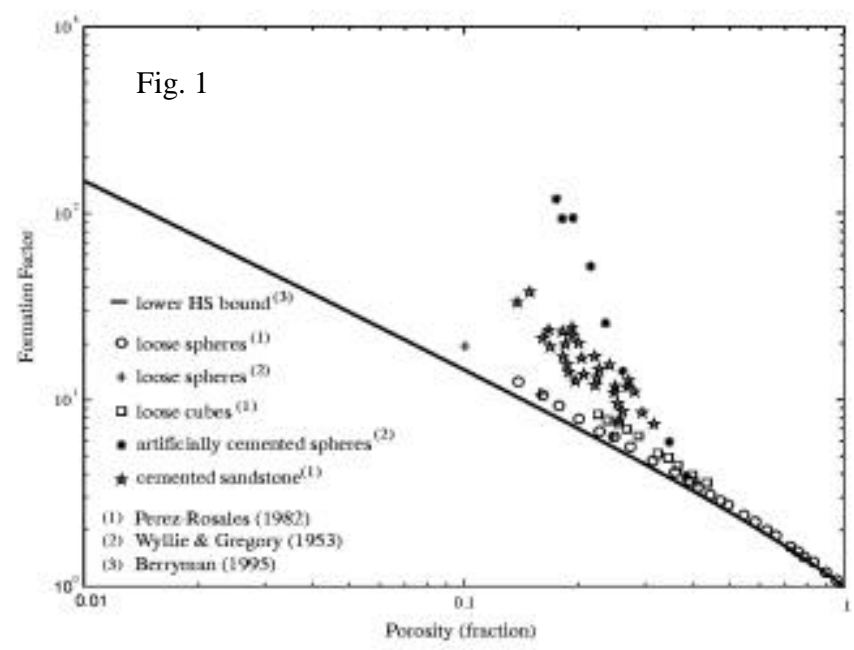

realized that accurate and reliable site characterization is the key to the success of any cleanup effort. Our research objective is to develop methodologies for inexpensive high resolution imaging of natural heterogeneities and for relating these heterogeneities to the hydrogeologic parameters that control flow and contaminant transport. Our focus is to help establish the scientific basis for applying shallow geophysics to hydrogeological problems, through sediment-rock physics. This includes identifying uncertainties in applying shallow geophysics interpretations to

hydrogeological site characterization.

The report summarizes work done since Spring 1999 related to the basic rock physics component of the project.

The objective of reservoir and aquifer characterization is to develop 3-D maps of flow properties so that fluid flow can be modeled. Historically, the petroleum industry has used geophysical data to predict flow

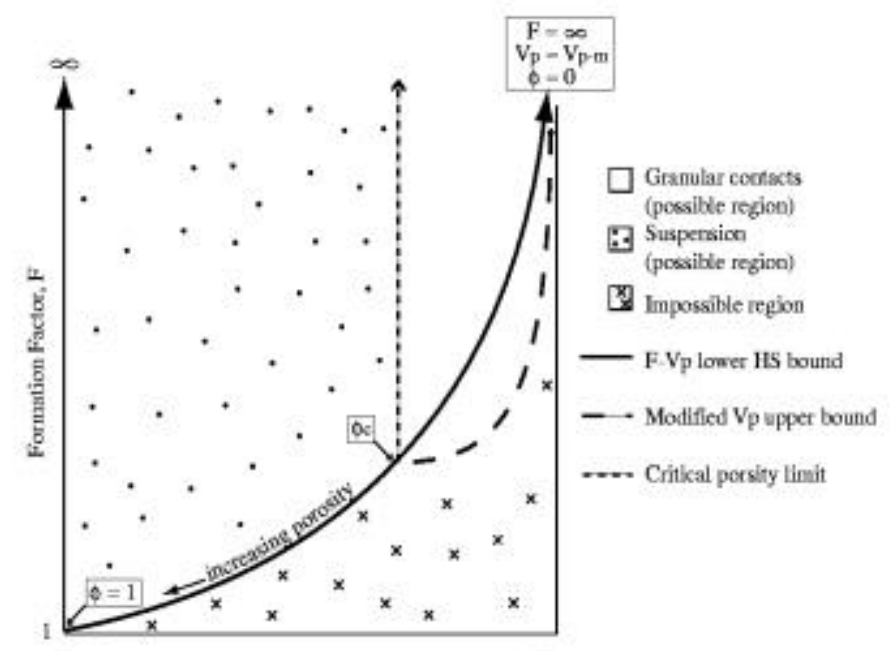


properties and develop such maps. Recently, attempts have been made to adapt the same techniques to shallow aquifer environments. In this report, we present a method for bounding the relationship between formation resistivity factor $F$ and compressional velocity $V_{p}$. This method constrains porosity by constraining possible ranges of $F-V_{p}$ data. Both the petroleum and environmental industries would benefit from this technique because of the potential for using electrical logs to better constrain seismic interpretations and develop more accurate maps of flow properties. Our approach is simple. It involves combining the well-known Hashin-Shtrikman bounds for electrical conductivity and acoustic velocity and tracking porosity changes with the bounds. The significance of our approach is in its potential for using known relationships between formation factor and material properties in sediments and rocks to explain velocity trends and vice versa. We found that $F-\phi$ data in unconsolidated glass beads, and suspensions (Perez-Rosales, 1982; Wyllie and Gregory, 1953) fall along the lower HS bound (Figure 1) and the $F-\phi$ values move up the lower HS bounds as a result of compaction and sorting while cementation moves the points away from the bound. Both resistivity and seismic velocity are significantly influenced by the same variables: pore structure (amount, texture and content), grain contacts (shape, sorting and cement degree and type) and clays (amount, type and distribution). Despite the complexity in the $F-\phi$ and $V_{p}-\phi$ relationships the electrical and acoustic Hashin-Shtrikman HS bounds can be combined at equal porosities to provide bounds on the $F-V_{p}$ relationship (Figure 2). The bold, solid line in Figure 2 represents the lower HS bounds and the curved, bold, dashed line represents the modified Voigt $V_{p}$ upper bound. The vertical dashed line represents a suspension porosity limit. No $F-V_{p}$ data is feasible within the region marked by x's. Thus combining electrical and elastic data narrows the feasible region, constraining the possible sediment properties.

Planned Activities: The final planned activities in this project which concludes in March 2000 include preparation of reports and manuscripts for publication.

Information Access: This work was presented at the SAGEEP 2000 meeting. It will also be available at the web site: http://water.stanford.edu/doe/doeEMSP2000.html 